\title{
The Design of a Flexible Hypermedia System
}

\author{
Integrating an Interactive Learning paradigm for Foreign \\ Language Text Comprehension
}

\author{
Alfredo Fernandez-Valmayor ${ }^{1}$, Covadonga Lopez-Alonso ${ }^{2}$, Arlette Sere $^{2}$, and \\ Baltasar Fernandez-Manjon ${ }^{1}$ \\ ${ }^{1}$ Escuela Superior de Informatica: ${ }^{2}$ Facultad de Filologia, Universidad Complutense de Madrid, \\ 28040 Madrid, Spain: \{alfredo, balta\}@sip.ucm.es: \{alonsolo, serebaby\}@eucmax.sim.ucm.es
}

Key words: Hypermedia design, SGML, language learning technologies, interactive educational environments, educational software development

\begin{abstract}
In this paper we discuss an approach to hypermedia system design based on learners' background knowledge and its dependency on the subject to be taught. Markup languages in general, and SGML in particular, play a central role in our approach. Using SGML as a meta-language, we can formalize, flexibly, the logical structure of the learning environment foreseen by designers. Design documents, produced accordingly to this SGML formalized structure, are the common workspace for both pedagogical designers and programmers
\end{abstract}

\section{INTRODUCTION}

The integration of new information and communication technologies offers a powerful synergy. It has the potential to provide a whole range of educational applications embodying new paradigms for learning [Ibrahim and Franklin 95, Wynblatt et al 97]. Today, many institutions are trying to integrate these technologies into current curricula. However, the current situation shows a big gap between educator's perceptions of the high potential of hypermedia as a new educational technology and its real use [Nunes and Fowell 96]. Even if hypermedia facilitate processes that are vital 
for learning (i.e., association and exploration), new media do not, just by themselves, guarantee learning [Hogan-Brun and Whittle 98].

We identify three different approaches to educational hypermedia design, depending on which components of the learning environment authors stress more: the content, the media or the learner. The first approach focuses on the content of the educational domain [Pimentel et al 98, Isakowitz et al 95]. Designers articulate the educational domain into courses, lessons, exercises, and quizzes. The content or, more specifically, a content model is central to this approach and design is usually based on a data base model (e.g., entityrelationship). This model is conceived as a theoretical framework that supports the task of structuring the educational domain. The provision of tools supporting the creation and manipulation of course structure is one of the main goals of this approach (see papers on curriculum development in this publication). Specific problems about the media and about learners' needs also arise in this approach, but they are not the central concern.

The second approach is based on a hypertext or hypermedia model [Hardman et al 94, Halasz and Mayer 94]. The central idea is to model an educational domain as a network of components, with links and anchors relating all of the identified components. The focus is on the media model: a basic node/link network structure that is considered the essence of a hyperapplication. Designers start building the application by trying to map the components of the educational domain onto components (atomic or complex) of the model. Design "focuses on the mechanisms by which link and non-link components are glued together to form hypertext networks" [Halasz and Mayer 94]. The granularity of the decomposition is, in this approach, a major design decision. Presentation specifications and synchronization of the different media involved are also important. The content of the course is adapted to this schema, as also are learner interactions that are mainly conceived as navigational decisions.

A third approach is centered on learner and learner needs. This is the approach that we consider more promising and that, in our opinion, better supports the construction of educational environments embodying new paradigms for learning. In this approach, design starts with the analysis of learners' previous knowledge about the topic and their potential interactions with the learning environment. From this perspective, designers identify the logical structure of pedagogical interactions and the hypermedia application is built taking into account the identified characteristics of the learner and the anticipated learning processes.

Our work uses this third approach, integrating an established learning paradigm for foreign language text comprehension, with a flexible methodology for building an electronic educational environment supporting this paradigm. This learning paradigm and its implementation as a 
hypermedia system is the result of the joint research effort of experts from two very different fields: language and computer specialists. The approach described in this paper has been developed as part of our work in the design and development of the hypermedia modules of a European project now in the evaluation and final delivery stages: "Galatea: Apprentissage de la Compréhension en Langages Voisines" [López, Alonso and Séré 97, 98, Fernandez-Manjon and Fernandez-Valmayor 97].

From the educational software production perspective, we have attained the flexibility required for our joint effort by using SGML (Standard Generalized Markup Language) [Golfard 90]. SGML concepts have been a critical element in the development of our hypermedia system and SGML has been used at both the design and the development phases of the project. Marked-up documents are the common workspace for both educators and programmers. In this way, for example, educators can produce and modify the marked-up documents using text-processing tools and programmers can use these marked-up documents as the input data for the hypermedia system that recreates at the computer the learning environment envisioned for educators [Fernandez-Manjon and Fernandez-Valmayor 98].

In this paper, we describe and analyze in detail the foundations and didactic dimensions of the learning strategies on which our application is based. Then we present how we are using markup languages to ease the description, construction, and maintenance of the hypermedia model of the previously analyzed learning environment. Finally, we discuss the main outcomes of the Galatea project and our future work on a new project.

\section{LEARNING TEXT COMPREHENSION IN A FOREIGN LANGUAGE}

Text comprehension in a foreign language is a complex activity, that demands qualitatively different levels of analysis: cognitive, linguistic and, social [Rastier 87, 91, Kintsch 82]. It also implies a high degree of interdisciplinary cooperation if we want to achieve a satisfactory explanatory theory as well as set of pedagogical clues and directives to be used in the development of an educational application. At least three factors can be identified in the construction of sense: a) strictly linguistic factors which explain the features of the text, b) cognitive and mental operations built by the learner from what is said in the text, and c) learner's general knowledge. Three parameters are also involved in the reading of a text: writer, text, and reader (or learner), where the text/reader pair is particularly important, since the reader's task is to reconstruct what is said in the text. To discover the writer's intentions relies, to a large extent, on the reader's linguistic 
knowledge that will enable him to reach the content of the text. We introduce briefly this text/reader paradigm and comprehension/language typology to finally focus on our identified criteria of readability that are fundamental to the design of our hypermedia model.

\subsection{The text/reader paradigm}

The text is both learning and reading object, since the reader never gives up reading the text. As Ricoeur [1976] states: "first is to be in the world, then to understand, then to interpret, then to say". Thus, we base the term "comprehension" and the term "interpretation" on this semiotic approach, and see them as two stages of the same trajectory, where comprehension is the process, and interpretation is the result. However, interpretation becomes particularly complex in a foreign language because, although readers make hypotheses about the intention of the text, the resulting interpretation depends not only on their linguistic knowledge, but also on their knowledge and comprehension of the culture associated with the foreign language. Since language and culture are inseparable, to read a text in a foreign language means to participate in other world representations and to embrace other knowledge.

\subsection{Comprehension as paraphrase}

The aim of reading a text in a foreign language is, as in reading a text in one's mother tongue, to guess the intention of the text. However, in the former, access to the text is not immediate because the reader is a learner of a new language (L2). Comprehension of an L2 text implies acquiring new know-how, where the linguistic and cultural knowledge of the mother tongue (L1) is involved, as well as a process of awareness of both the language and culture of L2. The process of interpretation is close to paraphrase, to the extent that a given text $\mathrm{X}$ is, through interpretation, formulated as other text " $X$ ", where a linguistic gap can exist between the two texts $X$ and " $X$ " [López Alonso and Séré 94]. As a consequence, text comprehension in a foreign language is a multiple process. Even if readers hypothesize the intention of the text, the resulting interpretation depends not only on their linguistic knowledge, but also on their interpretation of the culture and civilization associated with the foreign language. 


\subsection{Reader and language typology}

The construction of text meaning is determined by the reader's specific conditions, i.e., the reader's mother tongue and the language of the text. Strategies for reading comprehension are related to the potential similarities between the two languages, especially at first levels of learning. Despite having their own systems and features, two languages that pertain to the same type exhibit a set of common features, as well as a common metalinguistic reflection that is almost unconsciously activated in the first approach to the reading. In many cases, two languages of the same type also share a common system of beliefs and a pragmatic universe with similar communicative situations [López Alonso and Séré 97]. On the other hand, if the two languages do not belong to the same type and a connection between them cannot be established, the cognitive activity is not sufficient to enable comprehension. For instance, in the pairs Spanish/English, Spanish/German or Spanish/Dutch, vocabulary is not accessible to beginners, and thus they cannot activate an interpretative strategy relying on lexis.

This paper concerns inter-comprehension in Romance languages (which is the area encompassing our work in the Galatea project). When facing a foreign Romance language (L2), readers find numerous forms that can also be identified in their mother tongue (L1); in addition, readers tend to transfer the grammatical system of L1 to L2. The similarities between the two systems facilitate reading comprehension, helping readers predict and describe certain linguistic behaviors of transfer and interference. In other words, readers activate comparative strategies that allow them to use L1 in the process of reconstructing comprehension of L2. In this sense, we believe that this comparison between the two languages is the most efficient strategy available to readers in interpreting texts. Also, inference is undoubtedly the cognitive activity most used by readers to deduce the meaning of texts.

\subsection{Learning comprehension: criteria of readability}

As previously stated, when reading a text in a foreign language, readers use inference as their main strategy in building the meaning of the text. Inference is a mental process, which is activated through interpretation, consisting of a set of cognitive operations that allow readers (learners) to derive certain meanings from other meanings, either through deduction or induction. In foreign language comprehension, this activity demands a wider framework of study than the psychological frame, because readers constantly use their inferential ability at the contextual and co-textual level. Inference is drawn from the linguistic material as well as from the conditions resulting from readers' gaps of knowledge of the foreign language. In our 
observations, learners focus first on the text type, and, after that, they attempt to build sense. It is a strategy that starts at the global level and then shifts to the local; also, it is an interactive mechanism. Considering these facts and with the aim of reflecting the learning processes that takes place in text reading comprehension in typologically related languages, we propose four criteria of readability that go from the whole text to the sentence level [López Alonso and Sèrè 99, Van Dijk 80, 83].

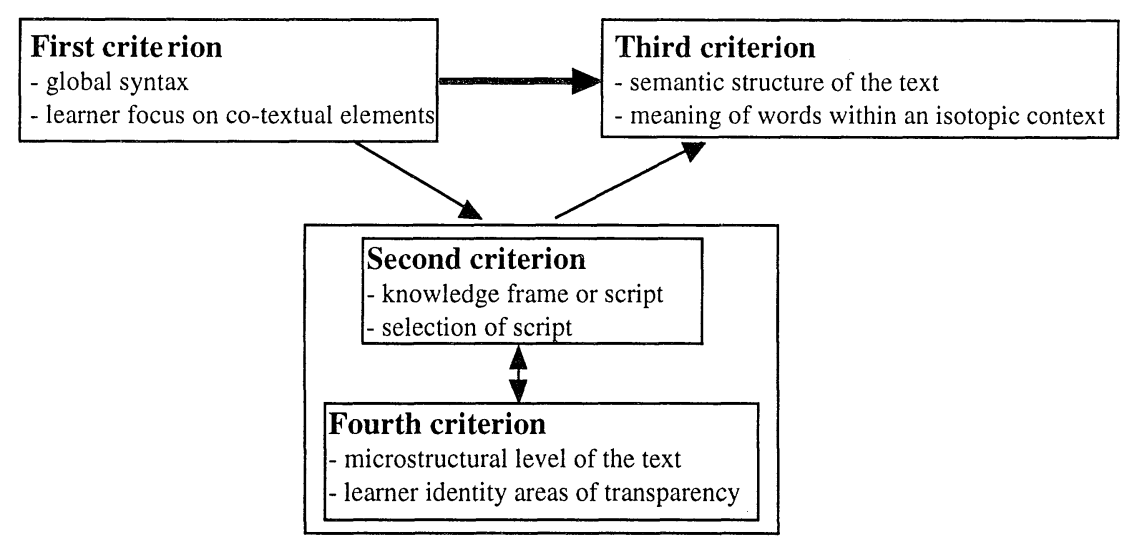

Figure 1. Comprehension strategies activated by readers of a language they do not know

\subsubsection{First criterion of readability}

The first criterion of readability lies in global syntax. Texts are classified accordingly to the representation that corresponds to its type. Identifying the type of a text, influences the interpretative strategies approached and the way meaning is comprehended. We conceive the text as an interpretative whole that bears both a pragmatic and a linguistic dimension [López Alonso and Sèrè 96]. Moreover, and despite the heterogeneity of texts, the reader always activates an abstract notion of text type. This abstract notion is an invariant that realizes the reader's representation of the text genre and triggers the first global meaning, which lies in the reader's textual competence. In our research, as mentioned, we have observed that readers of texts in foreign languages always establish an internal co-reference between the co-textual features and the cognitive schema implied by the text type. For this reason, genre is the first element that is activated in order to build meaning. 


\subsubsection{Second criterion of readability}

The second criterion of readability lies in the script or knowledge frame, which accounts for the representation of knowledge held by learners about the world, and that allows them to start the essential cognitive acts involved in language comprehension [Van Dijk 77]. Scripts are conventional and general and, in this sense, they correspond to a perception of the world and to a shared cultural and social universe. Scripts are also dynamic, and are therefore involved in plural interactions. From a linguistic point of view, the script emerges from a certain number of semantic propositions and of sentence functions that correspond to the text's macrostructure.

\subsubsection{Third criterion of readability}

The third criterion of readability lies in the semantic structure of the text, that is to say, in the macro-structural level of the text, which corresponds to its global meaning or to the set of semantic propositions that account for its microstructure. This level is essentially theoretical, but, as we shall see, it plays a basic role in retrospective inference. When texts in foreign languages are read, and especially in first levels of language learning, this level of readability must occur rapidly, because the subject often needs to contrast it with the lower levels of readability. When texts are read in one's mother tongue, this level is essential, because it ensures the interpretative coherence of the text. Conversely in foreign language texts, taking advantage of this level is more difficult, its role being rather that of supporting the interpretative hypotheses set by the readers.

\subsubsection{Fourth criterion of readability}

The fourth criterion of readability corresponds to the micro-structural level of the text. Even if the text is not just a simple addition of sentences, these sentences ensure the referential coherence resulting from the semantic sequencing obtained through an operation across sentences. In a foreign language, this level is essential, because it allows learners to acquire not only a competence of comprehension but also, and especially, a competence of expression.

We conclude that to read a text means to construct a mental representation of its invariance, to which multiple interpretative strategies are applied. In our research on the strategies used by readers facing a text in a language that they do not know, we have observed that the mental representations activated are, to a large extent, conscious. Readers relates the text type - first criterion of readability - with the possible concrete 
meaning it might bear - third criterion of readability. In order to build the latter, readers rely on the meaning of the words that can be inferred from its form - fourth criterion of readability. Thus, they can identify areas of transparency, while clarifying areas of opacity by making hypotheses from the contextual scripts — second criterion of readability [López Alonso and Sèrè 99].

\section{THE DESIGN OF A HYPERMEDIA APPLICATION FOR TEXT COMPREHENSION}

A well-founded and pedagogically sound learning environment is a critical stage in the production of educational software, because if the material is pedagogically weak, technology is not going to improve it. This is a first principle that implies much cooperative effort between educators and computer specialists. This is the approach we have followed and that can be traced to work previously done by other researchers [Bork et al 92]. However, in the construction of our application, we have relied on the flexibility that descriptive markup languages (i.e., SGML) offer to create a common workspace for both educators and programmers.

Descriptive markup is defined as "text that is added to the data of a document in order to convey information about it" [Goldfarb 90]. SGML provides not only a set of standardized codes that add meta-information to a document. SGML also provides a language that designers can use to define formally the structure of a whole class of documents. In SGML, this structure is formalized through a DTD (Document Type Definition) that declares the elements that compose the documents. A DTD is the grammar that formally describes the structure of a class of texts. (For example, HTML, the Hypertext Markup Language used in the Web is a specific application of SGML, and it can be considered as "the Web DTD"). A DTD describes the structure of documents in a way that is already familiar to linguists and designers of programming languages. It provides a notation to express a basic tree-like structure attaching a tag to each tree node. Tree nodes, tagged elements, can also be decorated with attributes expressing contextual relationships or any other kind of information we want to add to the document. SGML goes further, and we can also use a different document definition, the LPD (Link Process Definition), to associate processingoriented attributes formally with the structural elements (the tagged nodes of the tree) identified in the DTD. Using two kinds of documents (DTD and LPD), we have two different layers of application description. The advantage of having two layers is that we do not compromise the generality of our model description (DTD) with specific processing information (e.g., 
formatting). Moreover, the LPD makes it possible to associate different processing methods with the model depending, for example, on learner characteristics or on presentation devices. SGML also provides other means to make transparent to educational designers the more specific implementation problems, such as the physical decomposition into entities of the computer modules or its system processing dependencies. All such technical details about SGML are beyond the scope of this paper, but can be found in [Goldfarb 90].

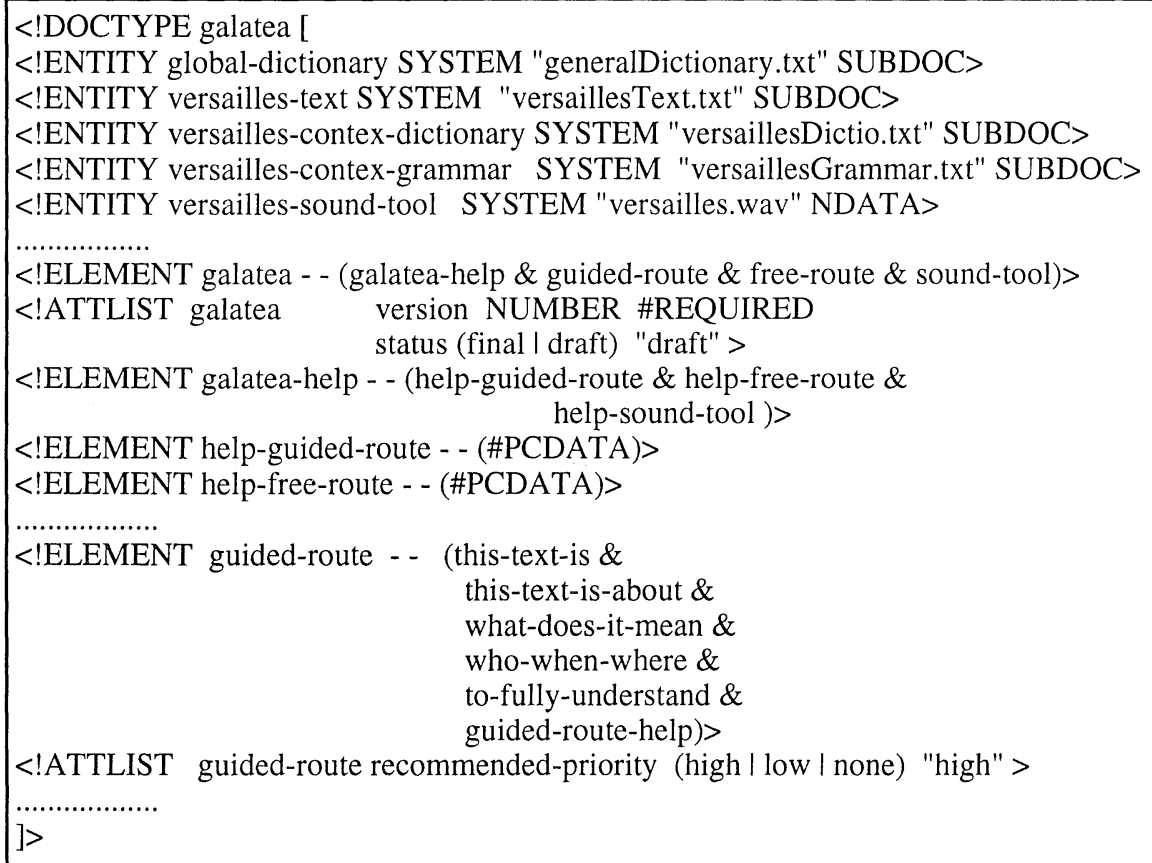

Figure 2. Partial DTD of the Galatea model

We use SGML facilities to describe formally the model of the application agreed upon by educators and computer specialists. DTDs and LPDs definitions are the common tools used by both kinds of specialist to build the educational application model. The elements that compose the application are basically organized in a simple tree-like structure. Attributes can express relationships between elements, characteristics of the element, or possible learner interactions. Based on the results described in the previous section, we designed a DTD that formally describes a learning environment aimed at teaching text comprehension (narrative-descriptive texts for readers of closely related languages). Based on this DTD, we have built a hypermedia application mainly addressed to beginners. In spite of the fact that here we 
describe the design process in a top-down manner, from the DTD to markedup documents, it must be clear that in "real life" we started working with marked-up documents in a bottom-up manner and afterwards, combined both directions.

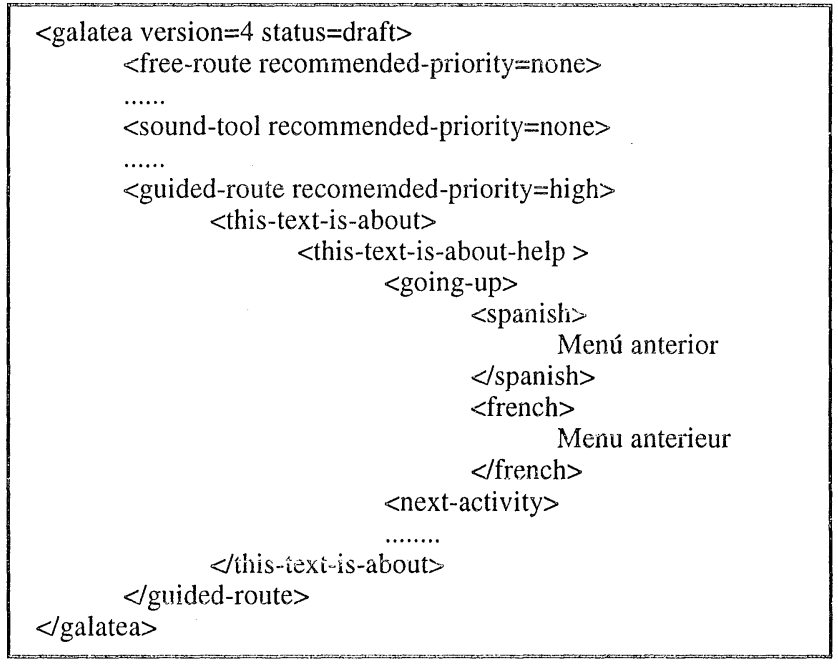

Figure 3. Marked documents are meaningful for both designers and application programs

In our application, we have applied the same DTD, the same model of learning environment to five different texts. Using the "subdocument" feature of SGML, we also include in the model the DTD of a narrativedescriptive text, the DTD of a contextual dictionary, the DTD of a contextual grammar, and the DTD of a general dictionary. The SGML "subdocument" feature allows us to incorporate separately created documents of various types (each of them with its own DTD) into a single document. In the following explanation, we focus on the model of the learning environment that is described in the main DTD.

\section{THE GALATEA MODEL}

The first level of the main DTD describes the first level of the learning environment. This is composed of four elements that can be considered in any order by the learner. One element is a tutorial to help readers to navigate through the course. Another element is listening, which allows readers to follow a recitation of the text. The other two elements are two kinds of pedagogic routes, which correspond to different learning strategies. One is a 
guided route, which includes different sets of activities organized in order, even if the order is not compulsory for the learner, and the other is a free route, where the learner can work freely through two contextual tools: a contextual dictionary and a contextual grammar. The two pedagogical routes are complementary and non-repetitive; however, a pedagogic approach would recommend starting with the guided route. This guided route is where most of the pedagogical design effort has occurred.

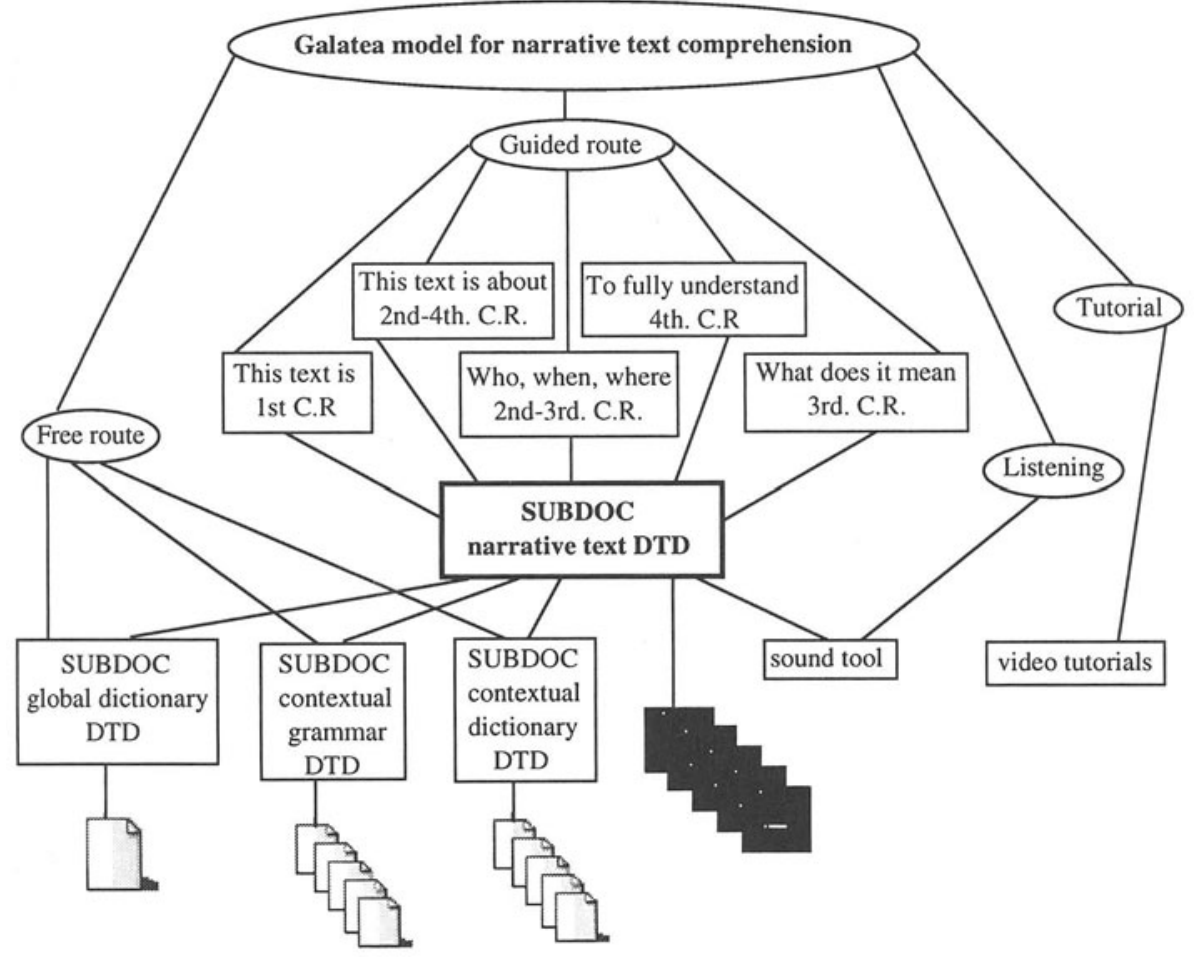

Figure 4. Graph representing the DTD with the general structure of the Galatea model

The first goal of a DTD is to provide a formal way to assign tags to the nodes of the tree that represents the logical structure of our model. After that, we can also add attributes to nodes, break the model into different entities, and so on. Consequently, when designing we can work with a tree-like representation of our model that afterwards it will be formalized by specialist as a DTD to which will be added all the details. Many of them will be always transparent to educational designers.

In figure 4, we can see the graph representing the general structure of our model. This graph represents the pedagogical interpretation we have made of the comprehension strategies activated by readers. The subdocument DTD 
describing the logical structure of the text (readers are working on) has a central position: "the reader never gives up reading the text" as stated in the text/reader paradigm. The guided tour, the stronger pedagogical approach to the reading of the text, is divided into five elements, each of them materializing as learners' activities and as learners' interactions with the text encompassing the four criteria of readability.

\subsection{Guided Route}

This text is...: Its aim is to activate the macro-processes involved in comprehension, and to help learners focus on the co-textual elements (first criterion of readability) which can lead them to make a first hypothesis about the meaning of the text. It includes three activities: identifying the text type, identifying typical elements, and placing the genre of the text.

This text is about...: This element focuses on the second criterion of readability and is also aimed at activating the macro-processes that will enable readers to elaborate the global meaning of the text. A first activity lies in lexical transparency between the two languages (fourth criterion of readability), and consists in the search for key words; a second activity proposes the selection of a storyboard representing the global meaning of the text (second criterion of readability).

What does it mean?: This element corresponds to the third criterion of readability; it is designed to work out the meaning of formally opaque words within an isotopic context. Isotopic networks (i.e., words belonging to the same semantic family) are marked in the text, while on the right of the screen the same networks appear as a list of words to be translated. The double graphic presentation helps learners to work simultaneously on two different levels of context: 1) the isotopic context of the lists (textual coherence), and 2) the phrastic context in the text (propositional coherence). Learners can choose the isotopic words that interest them and solve their queries by accessing the contextual dictionary.

Who, when, where?: This set of activities also relates to the third criterion of readability and is aimed at working more extensively on macro-processes; in particular, its purpose is to identify scripts (second criterion of readability). The scripts and their hierarchies vary among the texts. In the first text, for instance, they correspond to characters, time, and space. The first activity asks the learner to fill in a form about the characters. Then, two activities about the time script follow: 1) to identify the storyboard that describes the text, and 2) to organize the narrative events in chronological order. Finally, the space script is treated through a brief situation of the place where the event takes place; further, more general cultural elements are also offered. 
And to fully understand...: This set of activities refers to the fourth criterion of readability, and aims to clarify opaque areas of the texts. Two types of activities are offered: 1) a selection of true-false propositions, and 2) a selection of translations which correspond to areas of particular difficulty.

The DTD formalizing the content of the elements that compose the application states the model structure we have described in the previous paragraphs. Its interest is not only theoretical. The DTD is the definition to which marked-up documents, produced by pedagogical designers, must conform. On the other hand, programmers also build the interpreters of marked-up documents following the DTD (and the LPD). In this way, during the development phase, the testing and refining processes are greatly facilitated. When testing the prototypes, educators can modify the content of a marked document (a text file, figure 3), and the changes are automatically reflected in the application without any programming. For this task, standard text processing tools can be used, although it is clear that specialized tools could improve the process. Text files with marked-up documents can be opened during the development process, but they are codified for the final delivery version.

\section{FINAL REMARKS AND FUTURE WORK}

The issues we have discussed in this paper have evolved as a result of our work in the Galatea project. For that reason, the hypermedia model we have presented here corresponds not to the methodology we followed when we started to design and built the system, but to the conclusions we reached as a result of our work in the project. To develop our application, we initially chose an authoring system, but problems communicating with designers and maintaining prototypes led us to start working with marked up documents, initially with dictionaries and grammars. Then, we realized that having a marked-up document describing the whole pedagogical strategy of the application could be the nuclear component of the whole development process. Hence, we started to set up our application's DTD.

For practical reasons, the Galatea application still depends of a kernel built up using an authoring system that calls an interpreter that follows the DTD. Our future work will take another approach. We are now starting a new project that we intend to be platform and system independent. That means that the interpreters will be coded in Java with no dependencies on any authoring system. The small prototypes we have already done, running fragments of the Galatea environment, look promising. In this new project, we will also consider the potential advantages of using more specific standards derived from SGML, such as XML and HyTime, which could 
simplify the distribution and/or use of the application through the Internet. We will also plan to test the validity of our approach, extending our DTD to cover other types of texts (e.g., argumentative texts) and different pairs of languages such as English/Spanish. Finally, although working with mark-up languages has the advantage that designers can use standard text-processing tools it is clear that specialized editors could facilitate greatly the design process. The small prototypes already made look promising, and we plan to extend them in the new project.

Finally, it is also important to note that SGML has recently been used as a specification tool in all three approaches described at the start of this paper. SGML has been used to formalize an entity-relationship model for the educational domain [Pimentel et al 98, Isakowitz et al 95]. SGML has also been used to describe formally the network of components, anchors and links of the storage layer of Dexter and Amsterdam hypermedia models [Hardman et al 94, Halasz and Mayer 94]. The difference in the approach we have proposed relies, using SGML terminology, on the documents formalized and on the SGML resources used. In our approach, we first analyze in depth a learning situation (i.e., foreign language text comprehension) and then we use SGML to formalize at different levels of abstraction the documents that describe that learning situation. This situation could be very different, depending on learner characteristics (e.g., students learning a language that is closely related to their mother tongue) or depending on the subject or material to be taught (e.g., history or mathematics). We do not try to impose a preconceived model: partially structured network, or an entity-relationship model. However, it is also clear that the application of SGML has technical advantages in all three approaches; SGML is not a proprietary system, it provides platform independence and it allows the use of standard tools.

\section{ACKNOWLEDGEMENTS}

The EU project Galatea (TMI-LD-1995-1-FR89) and the Spanish Committee of Science and Technology (TIC97 2009-CE and TIC98-0733) have supported this work.

\section{REFERENCES}

Bork, A., Ibrahim, B., Levrat, B., Milne, A., Yoshii, R., 1992. The Irvine-Geneva Course Development System. Education and Society, Information Procesing 92. IFIP 12th Computer Congress Madrid, Spain 7-11 September 1992. Ed. North-Holland, Amsterdam. 
Fernandez-Manjon, B., Fernandez-Valmayor, A., 1997. "Improving World Wide Web educational uses promoting hypertext and standard general markup language contentbased features". Education and Information Technologies, vol 2, no 3, pp. 193-206.

Fernandez-Manjon, B., Navarro, A., Cigarran, J., Fernandez-Valmayor, A., 1998. "Using Standard Mark-Up in The Design and Development of Web Educational Software". En Davies, G., Wibe, J. (eds) Proceedings of Teleteaching 98, 5th IFIP World Computer Congress, Book series of the Austrian Computer Society

Goldfarb C.F., 1990. The SGML Handbook, Oxford University Press.

Halasz, F., Mayer, S., 1994. The Dexter Hypertext Reference Model. Communications of the ACM, 37(2), 30-39.

Hardman, L., Bulterman, D.C.A. and Van Rossum, G., 1994. The Amsterdam Hypermedia Model: Adding Time and Context to the Dexter Model. Communications of the ACM 37(2), 50-62.

Hogan-Brun, G., Whittle, R., 1998. The Potential of Multi-Media for Foreign Language Learning: A Critical Evaluation. Computers and the Humanities, 31, pp 451-457.

Ibrahim, B., Franklin, S.D., 1995. "Advanced Educational Uses of the World Wide Web". Computer Networks and ISDN Systems, vol 27, no 6, pp. 871-877.

Isakowitz, T, Stohr, E. A. and Balashumanian, P., 1995. RMM: A Methodology for Structured Hypermedia Design. Communications of the ACM 38(8), 34-44.

Kintsch, W., (1982 ) Aspects de la compréhension de textes, in Le Ny and W. Kintsch (Eds) Langage et Compréhension, Bulletin de Psychologie, 35, 777-787

López Alonso, C y Séré, A., (eds.) (1994) Langage, Théories et applications en F.L.E. Texte et compréhension, Madrid, SGEL.

López Alonso, C., Séré A. (1996) "Typologie des textes et stratégies de la compréhension en L.E."Etudes de Linguistique Appliquée, Didier-Érudition, pp. 441-451.

López-Alonso, C., Séré A., 1997. La construcción del sentido en lengua extranjera: contexto e inferencia, Estudios de lingüística, Henriquez, M.C y Esparza, M.A. (eds) Vigo, Dpto. Filología Españóla, págs. 139-154.

López-Alonso, C., Séré A., 1998. L'illusion de la forme du mot: opacité et transparence, De la didactique des langues 'a la didactique du plurilinguisme, Grenoble, CDL-LIDILEM

López Alonso, C, Séré, A (1999) "Discurso y texto: los procesos interpretativos”, Actas del I Simposio Internacional de Análisis del Discurso, edición CD-Rom, Universidad Complutense

Nunes, J., Fowell, S., 1996. Developing Educational Hypermedia Applications: A Methodological Approach. Information Research: an electronic journal, vol 2, no 2, October.

Pimentel, M.G.C., Santos, J.B., Fortes, R.P.M, 1998. Supporting Structured Teaching Material in the WWW. Journal of Universal Computer Science, vol. 4, no. 11, 825-838.

Rastier, F. 1987. Sémantique interprétative, París, PUF.

Rastier, F. 1991. Sémantique et recherches cognitives, París, PUF.

Ricoeur, P (1976) Interpretation theory. Discourse and the surplus of meaning, Texas Christian University Press

Van Dijk, T.A and Kinsch, W (1983) Strategies of discourse comprehension, New York, Academic Press

Van Dijk, T.-A (1977) Semantic macro-structures and knowledge frames in discourse comprenhension in M.-A Just y P.-A Carpenter (Eds.) Cognitive Processes in comprehension, Hillsdale, N.J., Erlbaum, 3-32

Van Dijk, T.-A (1980) Story comprehension: an introduction, Poetics 9, 1-21 
Wynblatt, M., Benson, D., Hsu, A., Bretshneider, F., Schessel, L., Howard, G., 1997.

Multimedia Meets the Internet: Present and Future. Multimedia Tools and Applications 5 , pp 7-32. 